\title{
High Value Chemosensors in the Era of Industrial Internet: Innovations in Materials, Transducers, and Data Analytics ${ }^{\dagger}$
}

\author{
Radislav A. Potyrailo \\ GE Global Research, 1 Research Circle, Niskayuna, NY 12309, USA; potyrailo@ge.com \\ + Presented at the 5th International Symposium on Sensor Science (I3S 2017), Barcelona, Spain, \\ 27-29 September 2017.
}

Published: 30 November 2017

Modern monitoring scenarios of gases for industrial safety and productivity, medical diagnostics, environmental surveillance, and other Industrial Internet applications demand new high value sensing capabilities of better accuracy, lower power consumption, and unobtrusive form factors. This talk will provide a critical overview of developments of a new generation of chemical sensors based on radio frequency identification (RFID) transducer platform and multivariable response principles to meet these new demanding sensing requirements. Multivariable sensors provide several partially or fully independent responses from an individual device to allow quantitation of several individual components in mixtures, rejection of interferences, and correction for environmental instabilities. Such multivariable sensors have been developed for monitoring of trace levels of toxic gases and environmental pollutants. We will discuss the design criteria of these sensors and the key roles of sensing materials and data analytics to achieve desired sensor performance. These developed multivariable sensors are attractive when selectivity advantages of classic off-line analytical instruments are cancelled by requirements for no consumables, low power, low cost, and unobtrusive form factors. We will conclude with a perspective for future needs in fundamental and applied aspects of chemosensing and with the 2025 roadmaps for ubiquitous gas monitoring.

(C) 2017 by the authors. Licensee MDPI, Basel, Switzerland. This article is an open access article distributed under the terms and conditions of the Creative Commons Attribution (CC BY) license (http://creativecommons.org/licenses/by/4.0/). 